\title{
INFORMES
}

\section{SESIÓN INAUGURAL DE LA CONFERENCIA REGIONAL LATINOAMERICANA DE POBLACIÓN}

\author{
(México, D. F., 17 de agosto de 1970)
}

\section{Palabras del Sr. Victor L. Urquidi, Presidente de El Colegio de México}

Damos hoy principio a una reunión que muchos no vacilarán en calificar de la mayor importancia en relación con el porvenir del hombre latinoamericano, pues ella trata del hombre mismo en su expresión numérica, su dinámica, su movilidad y otros muchos aspectos que conforman la estructura y características de una población. Es el hombre latinoamericano -estudiado a través de métodos científicos de análisis demográfico, social y económico- el que hoy precisamente nos concierne; el hombre que suma ya más de 280 millones y que puede llegar a más del doble, o sea 650 millones, para el año 2000 , cuando constituirá el 10 por ciento o más de la población mundial.

A los 300 demógrafos y otros especialistas, así como a los numerosos observadores, que vienen a tomar parte en el estudio y la discusión de estos conceptos, me es grato ofrecer, a nombre de las organizaciones coauspiciadoras, la más cordial bienvenida y desearles placentera y provechosa estadía en México, país que los acoge con su sincera hospitalidad de siempre.

Es mucho lo que se ha investigado sobre la población latinoamericana en los últimos años. Testimonio de ello son los más de 170 trabajos que se presentan a esta conferencia, trabajos que permitirán esclarecer $\rightarrow$ poner en duda, según el caso- importantes aspectos tocantes a mortalidad, fecundidad, migración y tendencias futuras, así como a las interrelaciones del crecimiento demográfico con el desarrollo económico y social. Con base en estos análisis, se podrá también debatir sobre política de población, asunto que está cada día más en la conciencia de los pueblos y los gobiernos de muchas partes del mundo. Y de todo ello saldrán sin duda propuestas para promover aún más la enseñanza y la investigación demográficas en nuestros países.

En el último cuarto de siglo no se había producido una conferencia sobre población en que hubiera, como en la actual, tan abundante participación de latinoamericanos - de hecho, cerca de 200- junto con estudiosos de otras áreas que se interesan en la demografía de América Latina. A la Unión Internacional para el Estudio Científico de la Población, que en el ámbito mundial promueve el estudio de los problemas demográficos, corresponde la presente iniciativa, nacida de la Conferencia Mundial de Población celebrada en Belgrado en 1965, donde la aportación de los latinoamericanos fue ya apreciable. Esta primera conferencia regional en América Latina cuenta, por supuesto, con el coauspicio del organismo al que más debe el progreso reciente de la investigación demográfica en nuestros países: el Centro Latinoamericano de Demografía, que tiene su sede en Santiago de Chile. Por su parte, la Comisión Económica para América Latina, tras haber examinado en forma sistemática e iluminante los problemas del desarrollo latinoamericano, ha abordado recientemente el estudio del elemento demográfico implícito en el mismo. Y ha cabido a 
El Colegio de Míéxico, a través de su Centro de Estudios Económicos y Demográficos, donde en los últimos cinco años se ha desarrollado un modesto esfuerzo de investigación, colaborar activamente en la organización y realización de los trabajos preparatorios de la Conferencia.

Al anterior patrocinio debe agregarse la cooperación eficaz y entusiasta que nos ha brindado el Gobierno de México, por conducto de la Secretaría de Industria y Comercio, de la que depende la Dirección General de Estadística. Se ha contado también con el apoyo firme del Programa de Actividades en Materia de Población de las Naciones Unidas, así como del Banco Interamericano de Desarrollo, el Banco Internacional de Reconstrucción y Fomento, la Fundación Rockefeller, la Agencia Sueca para el Desarrollo Internacional, la Agencia para el Desarrollo Internacional de los Estados Unidos, el Population Council y otros organismos técnicos y financieros nacionales $\mathrm{e}$ internacionales. Todos estos organismos han hecho posible esta Conferencia, en particular al proveer los medios que han facilitado la asistencia de tan elevado número de participantes.

Pero haríamos mal en atribuir a los distintos organismos e instituciones mencionados el mérito de esta reunión. En realidad, la conferencia es posible - y se justifíca- porque la demografía está logrando en América Latina su mayoría de edad, transcurrido ya en gran parte un período de formación sistemática de especialistas bajo los métodos modernos de esta ciencia; de tal suerte que se cuenta ya en América Latina con un contingente numeroso de demógrafos, que se han especializado en distintas ramas del estudio de la población y han mostrado su capacidad para efectuar innovaciones y utilizar los instrumentos analíticos en función de una realidad propia que es en tantos aspectos distinta a la de otras regiones del mundo. Está quedando atrás, rápidamente, si no es que casi ha desaparecido, aquella época en que el conocimiento de nuestros fenómenos demográficos nos llegaba a nosotros los latinoamericanos exclusivamente desde los institutos y oficinas ubicados en el extranjero, donde los censos y las estadísticas vitales eran analizados con poca intervención de nuestros especialistas. Esto no significa en absoluto desestimar la colaboración científica venida del exterior acerca de la población latinoamericana - su historia, su presente y su futuro- ni la que han prestado entidades como el Instituto Interamericano de Estadística y los programas de las Naciones Unidas. AI contrario, dicha colaboración ha sido valiosa en todos los órdenes y el hecho de que en esta reunión sigamos contando con ella es prueba feliz de que América Latina no trabaja aislada y de que acepta las aportaciones científicas de otras áreas. Lo que deseo subrayar es que debemos reconocer ampliamente que el esfuerzo propio de mejoramiento de la información de base, la investigación y el análisis, apoyado en instituciones propias de la región, es lo que mejor garantizará a la larga una consideración correcta de las complejas implicaciones de la dinámica demográfica por parte de gobernantes y gobernados, tanto de nuestros países como del extranjero.

El surgimiento de la nueva demografía latinoamericana queda comprobado por los 113 trabajos de autores latinoamericanos - dos tercios del total- que han sido presentados a discusión. Estos trabajos fueron escritos en su mayor parte por especialistas jóvenes vinculados a instituciones de reciente formación, o cuyas actividades han recibido nuevo impulso. Los temas son tratados con base en metodologías apropiadas y demuestran con amplitud la competencia técnica de sus autores y su comprensión de los aspectos sociales y económicos específicos de la población de los países latinoamericanos.

No obstante, afirmar los resultados positivos de la investigación demográfica latinoamericana de los últimos años no quiere decir que no existan 
todavía graves lagunas que sólo se colmarán mediante mayor esfuerzo en la formación de demógrafos, mayores recursos destinados a la investigación y mejor preparación de los datos básicos. A esto hay que agregar la necesidad de que sean cada día más numerosos los especialistas de otros campos de las ciencias sociales -economistas, sociólogos, antropólogos, politólogos, historiadores- que se interesen por la demografía. Entre las áreas de información insuficiente sobre la problemática poblacional latinoamericana destacan la mortalidad y sus causas, y la incertidumbre acerca de las tendencias de la fecundidad -campos muy complejos cuyo adecuado conocimiento, a través de estadística o de encuestas, tiene un costo sumamente elevado. Los estudios sobre migración entre áreas rurales y urbanas, fenómeno tan violento en América Latina, están apenas en sus comienzos. Las investigaciones sistemáticas sobre la urbanización como proceso social y económico del desarrollo se iniciaron hace unos cuantos años y no han permitido aún llegar a conclusiones generales útiles para los gobiernos en su política de localización industrial y de crecimiento de las ciudades. $\mathrm{Y}$ en términos generales, escasamente empiezan a estudiarse las vinculaciones empíricas entre el crecimiento de la población e importantes variables y condicionantes del desarrollo, tales como la formación del ahorro, la tasa de inversión, la distribución del ingreso, la absorción de la fuerza de trabajo en el empleo productivo, y la movilidad y el desequilibrio sociales.

De nuevo cabe subrayar la importancia de esta Conferencia por cuanto lleve a conocer más a fondo estas necesidades de investigación, a fin de proporcionar orientaciones para los próximos años. En ello deberán entrar en juego tanto formulaciones teórico-matemáticas nuevas como investigaciones concretas al nivel nacional, regional y local.

Vivimos en una era en que la naturaleza y la magnitud de los problemas que afronta la humanidad han llevado a muchos al convencimiento de que es necesario establecer metas, y adoptar medios y procedimientos congruentes entre sí, útiles para alcanzar objetivos superiores de convivencia nacional e internacional. Con arreglo a distintos enfoques, producto de la diversidad de ideas, tradiciones y experiencias del hombre, se asigna a este proceso un término común: el de planeación económica y social. Sin embargo, no existe ni ha existido planeación demográfica. Antes bien, la población ha sido y es objeto de toda suerte de influencias en su número, distribución, composición, calidad y otras características, sin que para ello medie ninguna orientación científica. Desde los más lejanos tiempos históricos, la población ha sido afectada por una gran diversidad de elementos naturales y sociales, físicos y psicológicos, políticos y económicos, que han acabado en los últimos ciento cincuenta años por darle el perfil que ahora tiene y las perspectivas que dentro de ciertos márgenes se pueden prever para los próximos treinta años. No ha habido una política consciente respecto a la población futura. Los acontecimientos sociales, económicos y políticos son promovidos en supuesto beneficio del hombre futuro, pero sin poder prever cómo reaccionará éste ante los mismos, sobre todo en cuanto a su deseo de reproducirse y seguir poblando el mundo a determinado ritmo de incremento. Por otro lado, nadie puede pretender que se suspenda o detenga el desarrollo ni la manifestación de sus componentes en espera de que demógrafos, sociólogos y economistas efectúen todos los estudios necesarios y de que, a su vez, los científicos examinen los límites de los recursos naturales y tecnológicos. Se va más bien adquiriendo gradualmente mejor conciencia de ciertos problemas como ya ocurre en la actualidad; y se ejerce la acción, la mayor parte de las veces, en forma parcial y no siempre con acierto. A este respecto, la función de la investigación es permitir que los elementos activos de la sociedad, desde el individuo, la familia, el grupo y la comunidad, hasta los instrumentos de gobierno, 
actúen con más racionalidad y armonía, en vista de los objetivos que se persigan. Así, si el objetivo es el comúnmente aceptado de elevar, mediante la planeación, el bienestar medio de la población (lo que en sí es una relativa abstracción) no es lógico que se fije la atención en todas las variables menos una, la población. Esta, a plazo intermedio y largo, es una variable tan importante como las demás. El volumen y la estructura de la población pueden ser influidos a través de actitudes y comportamiento del individuo, la familia y la comunidad y aun por actitudes nacionales.

Sería por otra parte erróneo ocuparse únicamente de la población y desatender los demás elementos del cambio social y económico. El desarrollo económico y social es aspiración de todos y debe promoverse con máximo empeño, por los medios más efectivos y justos. La cuestión que se plantea, al tratar sobre población, es "cuántos" serán "todos" en un porvenir más o menos asequible y con qué rapidez se llegará a una meta de bienestar que se considere satisfactoria. Es evidente que se necesitan tanto el capital como el trabajo, y que es necesario determinar la capacidad de desarrollo junto con la capacidad de consumo de la población. Se debe considerar al individuo como productor y como consumidor, y como miembro de una comunidad que procura una vida mejor, rodeada de satisfacciones no sólo materiales sino también morales y espirituales.

En los últimos 80 años, muchas sociedades han demostrado, a veces sin proponérselo en su conjunto, marcadas tendencias a limitar y aun reducir los niveles de fecundidad, por importantes razones de orden familiar o social. Hoy día, en varias partes"del mundo, sobre todo en países de menor desarrollo, el volumen y la tasa de incremento de la población se consideran ya críticos. Los gobiernos de esos países, apoyados en demandas populares, han iniciado programas que permiten a los cónyuges, con pleno acceso a información fidedigna de carácter científico y la ayuda de medios educativos, tomar las decisiones que juzguen más adecuadas respecto al tamaño de la familia y el espaciamiento del número de hijos. En Améfica Latina se ha comprobado la existencia de la demanda pero se han emprendido programas todavía muy limitados tendientes a que la población tenga la oportunidad de satisfacer esa demanda y se informe siquiera de las posibilidades que la ciencia ofrece.

La perduración de muy elevadas tasas de fecundidad como las que prevalecen en América Latina es un problema que está adquiriendo rasgos cada vez más angustiosos en la encrucijada en que se encuentra la mayoría de nuestros países. Esta afirmación se hace no porque las latinoamericanas se consideren naciones sobrepobladas sino porque experimentan ya pronunciadas tasas de crecimiento de su población que no tienen precedente histórico en parte alguna del mundo, ante una perspectiva evidente de condiciones adversas, tanto internas como externas, para un rápido desenvolvimiento económico y social. En estas circunstancias, la planeación del desarrollo deberá tener en cuenta, de aquí en adelante, una política de población como una de sus partes integrantes.

Así lo evidencian consideraciones que se han hecho en múltiples reuniones internacionales. A diferencia de algunas de ellas, sin embargo, la Conferencia que hoy nos reúne no habrá de pronunciarse sobre la conveniencia de que se emprendan determinadas políticas de población. Este es un asunto que compete a cada comunidad nacional con arreglo a sus propias instituciones y características. Pero las aportaciones individuales de muchos participantes y las discusiones que se entablen serán de gran valor para contribuir a ampliar en América Latina el diálogo que ya se ha iniciado y que es necesario proseguir desapasionadamente en los próximos años, a fin de que se puedan precisar objetivos y fijar posiciones basadas en el conocimiento de la realidad y no en simples prejuicios o dogmas. 
La política de población deberá ser el resultado del análisis científico; no es cuestión de opiniones. Los demógrafos no deben eludir este tema fundamental, pero su función no es la de jueces ni tampoco la de dictaminadores, sino la de personas capaces de efectuar, a la luz de los datos básicos sobre la población y de las influencias recíprocas entre ésta y los fenómenos del desarrollo, el análisis más amplio posible de los problemas.

Permítaseme concluir haciendo hincapié en la necesidad de impulsar y mejorar aún más la investigación demográfica en América Latina, y la formación de los especialistas capaces de intervenir en ella. Hace cerca de medio siglo ya lo profetizaba el pensador peruano José Carlos Mariátegui:

¿Hace falta remarcar que un país que no conoce su demografía, tampoco conoce su economía? No se puede saber lo que un pais produce, consume y ahorra si se ignora esta cosa fundamental: la población. Todos los estudios, todas las previsiones... parten de este dato. El economista, el político... antes de formular cualquiera teoría, antes de propugnar cualquiera orientación, averigua el movimiento demográfico, su ritmo y su proceso... En un país donde no se puede contar a los hombres, menos aún se puede contar la producción. Se desconoce el primero de sus factores: el factor humano, el factor trabajo. ${ }^{1}$

Analicemos entonces el recurso humano, que no sólo es factor de la producción sino objeto en sí de toda la actividad productiva, y apliquemos a su conocimiento el rigor científico de los métodos de la investigación demográfica, social y económica de nuestros días.

\section{Palabras de la señorita Carmen a Miró, Directora del Centro Latinoamericano de Demografía (CELADE)}

Esta tribuna me ofrece en primerísimo lugar la oportunidad de dejar pública constancia del agradecimiento del Centro Latinoamericano de Demografía (CELADE) al gobierno de México que, con su apoyo, ha hecho posible la realización de esta Conferencia, que sin duda ha de constituir uno de los hitos en el desarrollo de la demografía en América Latina.

Nunca como en esta ocasión he cumplido con mayor satisfacción y orgullo el cargo de representar al CELADE. Satisfacción, porque nos permite destacar la constatación de que la demografía, como disciplina científica, ha entrado en una etapa de franca madurez en nuestra región.

Ahí están para atestiguarlo los documentos de esta Conferencia, en su mayoría preparados por latinoamericanos y que, en general, contienen aportes de valor para el conocimiento de nuestra realidad demográfica o para la aplicación de técnicas de análisis aptas para el estudio de los fenómenos de población.

Aquí está para rubricar nuestra madurez la presencia de tan selecto grupo de estudiosos de temas relacionados con la población. Y sobre todo, aquí está para confirmarnos en nuestro aserto, esta insigne institución: El Colegio de México, que a través de su Centro de Estudios Económicos y Demográficos ha enfrentado con éxito que nos mueve al aplauso, esta Conferencia, hecho que, además, compromete la admiración y gratitud de todos nosotros.

A la satisfacción que nos embarga, se agrega - lo dijimos al comenzarun cierto orgullo que emana - perdónesenos la inmodestia- de la sensa-

1 José Carlos Mariátegui, Peruanicemos al Perí. Colección de Obras Completas, Biblioteca Amauta. Lima, edición de 1970, p. 86. 
ción que tenemos de que en alguna medida la labor desarrollada por CELADE ha contribuido a alcanzar la madurez que hoy apuntamos. A más de 200 latinoamericanos que han pasado por sus aulas hemos insistido en hacerlos partícipes de esa mística que nos induce a estudiar con seriedad y responsabilidad esa parcela de nuestra angustiante realidad, que tan directamente toca a nuestros problemas de desarrollo: la población. Un grupo numeroso de ellos se encuentra hoy aquí y justo es reconocerles que laboran con tesón y dedicación para lograr que la enseñanza y la investigación demográficas se expandan y se eleven a niveles comparables a los alcanzados en regiones más desarrolladas.

Otras instituciones científicas, claro está, han contribuido de manera efectiva también a que avancemos hasta el punto en que ahora nos encontramos. Debemos, en primer lugar, subrayar que la Unión Internacional para el Estudio Científico de la Población, al propiciar la participación de latinoamericanos en sus asambleas y en sus comités, ha sido poderoso estímulo para que hayamos procurado hacernos dignos de la oportunidad que se nos brindó. La iniciativa de realizar esta Conferencia Regional está ya dando sus frutos, pero no hay duaja de que ella ha de tener un efecto multiplicador en los próximos años. A los personeros de la Unión aquí presentes, trasmitimos nuestros agradecimientos, con la promesa de que sabremos aprovechar las enseñanzas que de esta Conferencia emanen, para que el estudio científico de la población continúe ocupando cimero lugar entre nuestras actividades.

El Centro de Estudios Económicos y Demográficos de El Colegio de México, fundado hace apenas 6 años, no puede ya dejar de ser automáticamente incluido en la lista de las instituciones académicas, que en forma sistemática y de manera continua han dado su aporte al crecimiento de la disciplina que cultivamos. Su revista Demografía y Economía circula para llevar a todo el mundo el testimonio de nuestra cultura demográfica. $\mathrm{Y}$ esta Conferencia lo consagra como uno de los máximos promotores del desarrollo ascendente de los estudios de población en la región. A Víctor Urquidi, hombre de visión y creatividad, y a sus muy efectivos colaboradores, nuestro homenaje y nuestro agradecimiento.

Reconocemos el riesgo de omisiones en que se incurre cuando de enumeraciones supuestamente exhaustivas se trata, pero al CELADE lo unen vínculos de colahoración con organismos profesionales de la región que, justo es reconocer aquí, han contribuido, cada uno dentro de su esfera y en la medida de sus esfuerzos, a la madurez de que ahora nos enorgullecemos. El Instituto Di Tella, en la Argentina, la Universidad de los Andes, en Colombia, la Universidad de Costa Rica, la Escuela de Salubridad de la Universidad de Chile, el Centro de Investigaciones Sociales por Muestreo, del Perú, merecen ubicarse en lugar destacado por su persistente apoyo a la investigación demográfica. A estas instituciones se han agregado recientemente algunas universidades latinoamericanas que, como la de Córdoba, en la Argentina, y la de Zulia, en Venezuela, realizan esfuerzos por institucionalizar la enseñanza de la demografía en su seno.

Nada nos parece más apropiado para terminar esta enumeración, que traer a nuestro recuerdo la memoria venerable del demógrafo que más ha contribuido al desarrollo de lo que bien podríamos llamar la demografía latinoamericana: Giorgio Mortara. En su Laboratorio en el Conselho Nacional de Estatistica do Brasil, se elaboraron muchos de los métodos que hoy se aplican no sólo en América Latina, sino en otras regiones del mundo. Los que tuvimos la dicha y el honor de conocer al profesor Mortara, sabemos que él habría compartido hoy nuestra satisfacción y nuestro orgullo. Porque en CELADE hemos procurado siempre aprovechar sus enseñanzas y sentimos, por tanto, que tenemos contraída con él deuda de 
profunda gratitud, queremos rendir en esta ocasión, emocionado tributo a su memoria. Que el sentido común, la creatividad y el ingenio que constituyeron las características dominantes de su quehacer científico, presidan nuestros actos en la próxima etapa de expansión y consolidación de los estudios de población, que estamos seguros se iniciará a partir de esta Conferencia.

En esta nueva etapa, confiamos en que ha de acompañarnos también efectivamente la Comisión Económica para América Latina, como uno de los medios de asegurar que nuestra actividad no quedará reducida a un mero ejercicio metodológico, sino que significará un aporte fundamental en el esfuerzo de sacar a nuestros pueblos del subdesarrollo. 